
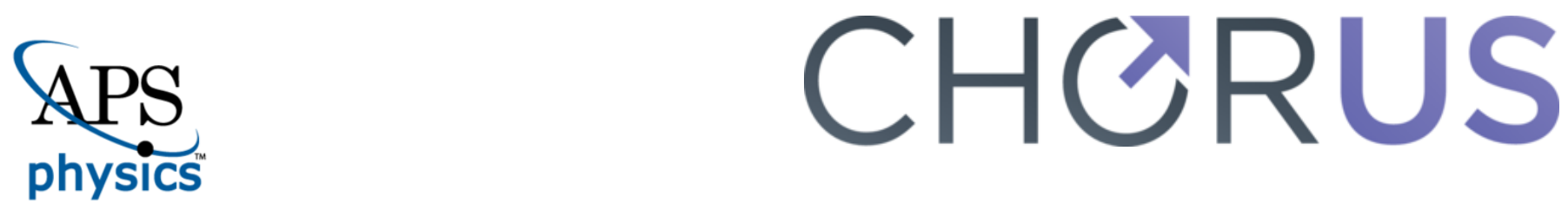

This is the accepted manuscript made available via CHORUS. The article has been published as:

\title{
Ultracompact Minihalos as Probes of Inflationary Cosmology
}

Grigor Aslanyan, Layne C. Price, Jenni Adams, Torsten Bringmann, Hamish A. Clark, Richard Easther, Geraint F. Lewis, and Pat Scott

Phys. Rev. Lett. 117, 141102 - Published 28 September 2016

DOI: 10.1103/PhysRevLett.117.141102 


\title{
Ultracompact minihalos as probes of inflationary cosmology
}

\author{
Grigor Aslanyan, ${ }^{1,2, *}$ Layne C. Price,,${ }^{1,3, \dagger}$ Jenni Adams,,${ }^{4,}$ Torsten Bringmann,,${ }^{5,}$ \\ Hamish A. Clark, ${ }^{6,}$ Richard Easther, ${ }^{1, * *}$ Geraint F. Lewis, ${ }^{6,}{ }^{\dagger \dagger}$ and Pat Scott ${ }^{7}$, \\ ${ }^{1}$ Department of Physics, University of Auckland, Private Bag 92019, Auckland, New Zealand \\ ${ }^{2}$ Berkeley Center for Cosmological Physics, University of California, Berkeley, CA 94720, USA \\ ${ }^{3}$ McWilliams Center for Cosmology, Department of Physics, \\ Carnegie Mellon University, Pittsburgh, PA 15213, USA \\ ${ }^{4}$ Department of Physics and Astronomy, University of Canterbury, Christchurch, 8140, New Zealand \\ ${ }^{5}$ Department of Physics, University of Oslo, Box 1048 NO-0316 Oslo, Norway \\ ${ }^{6}$ Sydney Institute for Astronomy, School of Physics A28, \\ The University of Sydney, NSW 2006, Australia \\ ${ }^{7}$ Department of Physics, Imperial College London, \\ Blackett Laboratory, Prince Consort Road, London SW7 2AZ, UK
}

(Dated: August 11, 2016)

\begin{abstract}
Cosmological inflation generates primordial density perturbations on all scales, including those far too small to contribute to the cosmic microwave background. At these scales, isolated ultracompact minihalos of dark matter can form well before standard structure formation, if the perturbations have sufficient amplitude. Minihalos affect pulsar timing data and are potentially bright sources of gamma rays. The resulting constraints significantly extend the observable window of inflation in the presence of cold dark matter, coupling two of the key problems in modern cosmology.
\end{abstract}

Introduction. - Observations of the cosmic microwave background (CMB) [1-3] provide firm evidence for the existence of dark matter (DM), as do astrophysical data on galaxy scales. The same experiments also show that inflation provides a robust account of the physics of the early Universe [4]. However, the microphysical bases of inflation and DM are unknown and require physics outside the Standard Model. The leading candidates for DM are weakly-interacting massive particles (WIMPs), which arise in many well-motivated theories Beyond the Standard Model. Conversely, inflation typically operates at energies near the scale of grand unified theories [5]. This Letter demonstrates that joint analyses of the DM and inflationary sectors yield tighter constraints than those obtained by treating each sector in isolation.

Dark matter and inflation are connected via primordial density perturbations at small physical scales, which arise from quantum fluctuations in scalar field(s) during inflation [6]. If the amplitude of fluctuations at small scales is significantly larger than at the scales of the $\mathrm{CMB}$ and large scale structure, ultracompact minihalos of DM (UCMHs) can form shortly after matter-radiation equality [7-9]. Recent limits on the UCMH abundance from astrophysical searches for DM annihilation [9-11] constrain the power spectrum at scales far smaller than those that contribute to the CMB. Limits from pulsar timing [12] are projected to lead to similarly strong constraints, and would have the added benefit of not requiring DM to annihilate. For even larger fluctuation amplitudes, primordial black hole $(\mathrm{PBH})$ formation is possible [13], leading to complementary constraints on inflation [14].

In this Letter we provide strong and robust limits on the shape of the inflationary potential and the primordial power spectrum by combining large-scale CMB data with small-scale constraints on the number densities of PBHs [15] and UCMHs [10, 12, 16]. This method allows one to simultaneously test standard inflation and the nature of DM, by cross-correlating the pulsar and $\gamma$-ray signals. We apply these constraints to a flexible model of inflation, which can reproduce the results of standard scenarios, e.g. chaotic [17], hilltop [6], and small-field inflation. Under very conservative assumptions, we find UCMHs provide comparable constraints on inflation to PBHs, but that they could be even more powerful probes of inflation if we could better understand their formation.

Ultracompact minihalos (UCMHs). - A UCMH, as opposed to a regular DM minihalo, collapses before some critical redshift $z_{\mathrm{c}} \gtrsim \mathcal{O}(100)$. These halos form in isolation, with extremely small velocity dispersions, via almost pure radial infall. This produces a steep density profile $\rho_{D M} \propto r^{-9 / 4}[8,18]$ with an inner plateau due to finite DM angular momentum $[9,10]$ and possible DM self-annihilation. This compact core makes UCMHs insensitive to tidal disruption [19]. Because annihilation scales with $\rho_{D M}^{2}$, they are excellent indirect DM search targets [9-11]. Time-delay lensing can constrain the UCMH number density, as a UCMH that passes near the line of sight between Earth and a distant pulsar would cause a change in its observed pulsation rate [12].

Assuming that UCMHs track the bulk DM density, both on cosmological and Galactic scales, limits on their cosmological abundance can be inferred from local limits on the UCMH number density. If DM annihilates, $\gamma$-ray limits from Fermi-LAT provide the strongest bounds [10]. The impacts of WIMP annihilation in UCMHs on reionisation may also be apparent in the 
CMB [20, 21]. Constraints from pulsars [12], based on gravitational effects only, would be entirely modelindependent [22]; while extending over a smaller range of scales, projected limits are at least as constraining as gamma-ray constraints. Complementary but weaker constraints can also be obtained from CMB spectral distortions [23, 24]. These limits constrain the processes that could have formed UCMH-seeding overdensities in the early Universe [10, 11, 16, 21, 25-28].

The fraction of DM in UCMHs with present-day mass $M_{0}$ is $f=\Omega_{\mathrm{UCMH}} / \Omega_{\chi}=\left(M_{0} / M_{i}\right) \beta(R)$ [10], where $M_{i}$ is the initial mass contained in an overdense region of comoving size $R$. For a Gaussian distribution, the fraction of perturbations that collapse to form UCMHs is

$$
\beta(R)=\frac{1}{\sqrt{2 \pi} \sigma_{\chi, \mathrm{H}}(R)} \int_{\delta_{\chi}^{\min }}^{\delta_{\chi}^{\max }} \exp \left[-\frac{\delta_{\chi}^{2}}{2 \sigma_{\chi, \mathrm{H}}^{2}(R)}\right] \mathrm{d} \delta_{\chi} .
$$

Here, the minimum density contrast $\delta_{\min }$ required for UCMH formation is the minimum amplitude at horizon entry that a perturbation must possess for it to have sufficient time to begin nonlinear collapse before $z_{\mathrm{c}}$. Typically $\delta_{\min } \sim 10^{-3}[8,10]$. If the initial overdensity is too large, $\delta_{\chi} \geq \delta_{\chi}^{\max } \sim \mathcal{O}(1)$, a $\mathrm{PBH}$ rather than a $\mathrm{UCMH}$ would form. However, since $\delta_{\chi}^{\min } \ll \delta_{\chi}^{\max }, \beta(R)$ is independent of $\delta_{\chi}^{\max }$ to a very good approximation. The quantity $\sigma_{\chi, \mathrm{H}}(R)$ is the mass variance of perturbations at the time $t_{k_{R}}$ of horizon-entry of the scale $k_{R} \sim 1 / R$. It is roughly proportional to the total size of perturbations at $t_{k_{R}}, \sigma_{\mathrm{H}}^{2}(R)=A_{\chi}^{2}\left(k_{R}\right) \delta_{\mathrm{H}}^{2}\left(t_{k_{R}}\right)$, where the factor $A_{\chi}$ depends on the initial spectrum of perturbations produced during inflation and the expansion history since [29]. In the special case of an almost scale-free spectrum with a spectral index $n_{s}(k)$ that runs only at first order [10],

$$
\begin{aligned}
A_{\chi}^{2}(k)=\frac{9}{16} \int_{0}^{\infty} d x x^{n_{s}\left(k_{*}\right)}+2+\alpha_{s} \ln \left(\frac{x k}{k_{*}}\right) & \left(\frac{k}{k_{*}}\right)^{\alpha_{s} \ln x} \\
& \times W_{\mathrm{TH}}^{2}(x) \frac{T_{\chi}^{2}(x / \sqrt{3})}{T_{\mathrm{r}}^{2}(1 / \sqrt{3})},
\end{aligned}
$$

where $W_{\mathrm{TH}}$ is the Fourier transform of a spherical top-hat window function, $T_{\mathrm{r}}\left(T_{\chi}\right)$ is the radiation $(\mathrm{DM})$ transfer function, and $\alpha_{s} \equiv \mathrm{d} n_{\mathrm{s}} / \mathrm{d} \ln k$ is the running of the spectral index $n_{s}$. However, inflationary models generally have a scale dependence beyond $\alpha_{s}$ and we therefore apply UCMH constraints using the local slope of the power spectrum instead, i.e. we set $\alpha_{s}=0$ and replace $n_{s}\left(k_{*}\right) \rightarrow n_{s}(k)$.

The most crucial non-primordial parameter for the UCMH abundance is $z_{\mathrm{c}}$, the lowest redshift at which collapse happens radially and in full isolation. Smaller $z_{\mathrm{c}}$ allows smaller-amplitude perturbations to form UCMHs, as perturbations have longer to collapse. This parameter is poorly constrained, as it represents the redshift at which the approximations of spherical collapse and secondary infall break down [18]. These are excellent approximations at $z \gtrsim 1000$, but when nonlinear structure formation begins at $z \lesssim 30$, these conditions certainly do not hold. In this Letter we use $z_{c}=1000$ as an extremely conservative choice, but show how limits improve with $z_{\mathrm{c}}=500$ and $z_{\mathrm{c}}=200$, which are both realistic possibilities.

Limits on the UCMH abundance.- Gamma-ray fluxes depend on $\rho_{D M}$, the DM mass $m_{\chi}$, annihilation cross-section $\langle\sigma v\rangle$, and annihilation branching fractions into different final states. Lighter WIMPs produce larger fluxes; we make the conservative choice $m_{\chi}=1 \mathrm{TeV}$. We assume an NFW profile for the Milky Way, the canonical 'thermal value' for the annihilation cross-section $\langle\sigma v\rangle=3 \times 10^{-26} \mathrm{~cm}^{3} \mathrm{~s}^{-1}$, and $100 \%$ annihilation into $b \bar{b}$ pairs (which produce $\gamma$-rays mostly by neutral pion decay). The limits are not especially sensitive to these assumptions $[9,10]$. We adopt the likelihood function of Refs. $[10,26]$ for the abundance of UCMHs indicated by Fermi-LAT $\gamma$-ray observations [30], based on the diffuse flux from the Galactic poles, and the non-observation of DM minihalo sources in the first year of all-sky survey data.

If $\mathrm{DM}$ does not annihilate, pulsars provide the only realistic means of detecting low-mass UCMHs. Here we apply the projected constraints from the individual-halo Shapiro delay detection method of Ref. [12], assuming a transit detection threshold of 20 ns. Assuming nondetection of UCMH transits within 30-year pulsar timing data provides the strongest projected gravitational bound on UCMHs with masses $\sim 10^{-3} M_{\odot}$. While the assumed detection threshold provides relatively weak limits on the fraction of DM contained within UCMHs compared to those from gamma-ray searches, it may soon be improved with the development of high-sensitivity pulsar timing arrays, improved understanding of the nature of pulsar timing noise, and increased observation time in existing millisecond pulsar surveys. The corresponding limits on the power spectrum only apply in the local vicinity of the scale $k_{R}$, i.e., where the predicted power spectrum is approximately locally power law. Although pulsar limits are weaker than $\gamma$-ray ones, they are purely gravitational, and would apply regardless of the precise particle properties of DM.

The observable window of inflation. - We use a phenomenological inflation model that can mimic many plausible scenarios, including large-field and small-field inflation, which have large and small values of the tensor-toscalar ratio $r$, respectively. We parametrize the inflationary potential as

$$
V(\phi)=\sum_{n=0}^{4} \frac{V_{n}}{n !}\left(\phi-\phi_{*}\right)^{n},
$$

where $\phi_{*}$ is the inflaton field value when the pivot scale $k_{*}=0.05 \mathrm{Mpc}^{-1}$ leaves the horizon, which is fixed to 
$\phi_{*}=0$ without loss of generality. The real constants $V_{n}$ are related to the slow-roll parameters $\left\{\epsilon_{*}, \eta_{*}, \xi_{*}, \omega_{*}\right\}$ evaluated at $\phi=\phi_{*}$ by

$$
\begin{aligned}
V_{1}=\frac{V_{0} \sqrt{2 \epsilon_{*}}}{M_{\mathrm{Pl}}} & \text { with } & \epsilon_{*}=\frac{M_{\mathrm{Pl}}^{2}}{2}\left(\frac{V^{\prime}}{V}\right)^{2}, \\
V_{2}=\frac{V_{0} \eta_{*}}{M_{\mathrm{Pl}}^{2}} & \text { with } & \eta_{*}=M_{\mathrm{Pl}}^{2} \frac{V^{\prime \prime}}{V}, \\
V_{3}=\frac{\xi_{*}^{2} V_{0}^{2}}{M_{\mathrm{Pl}}^{4} V_{1}} & \text { with } & \xi_{*}^{2}=M_{\mathrm{Pl}}^{4} \frac{V^{\prime} V^{\prime \prime \prime}}{V^{2}}, \\
V_{4}=\frac{\omega_{*}^{3} V_{0}^{3}}{M_{\mathrm{Pl}}^{6} V_{1}^{2}} & \text { with } & \omega_{*}^{3}=M_{\mathrm{Pl}}^{6} \frac{V^{\prime 2} V^{\prime \prime \prime \prime}}{V^{3}},
\end{aligned}
$$

and $V_{0}=V\left(\phi_{*}\right)$, where $M_{\mathrm{Pl}}^{2}=1 / 8 \pi$.

Expanding $V$ to fourth-order in $\phi$ allows the primordial spectrum $\mathcal{P}_{\zeta}(k)$ to have a running spectral index $\alpha_{s}$ and a higher order running-of-the-running $\alpha_{s}^{\prime} \equiv d \alpha_{s} / d \ln k$, giving significant freedom in the shape of $\mathcal{P}_{\zeta}(k)$, although this cannot easily replicate $V(\phi)$ with a step or sinusoidal oscillations. The potential (3) was used in Refs. [4, 31, 32 ] as an empirical description of the primordial epoch, constrainable in a CMB "observable window" of scales $10^{-6} \lesssim k / \mathrm{Mpc}^{-1} \lesssim 10^{-1}$. Measurements of the power spectrum put tight limits on the slow-roll parameters, ensuring the plausible domain of validity of (3) is larger than $M_{\mathrm{Pl}}$, and therefore describes the potential through $\mathcal{O}(10-100) e$-folds of inflation. Furthermore, (3) is the minimal polynomial potential for which $n_{s}, \alpha_{s}$, and $\alpha_{s}^{\prime}$ are independent and potentially non-trivial.

Using the ModeCode inflation package [33], we solve the equations of motion for $\phi(t)$ and the perturbations $\delta \phi(t, k)$ numerically, assuming the Bunch-Davies initial condition on sub-horizon scales [34]. We do not require slow-roll to hold during inflation or $V>0$ except at $V_{0}$, since inflation must end before $V<0$. We also include results using the inflation module from Class [35], which replicates previous techniques $[4,31,32]$. We find no difference between the two implementations where they overlap.

For fixed $V_{n}$ the number of $e$-folds $N_{*}$ between horizon exit for the pivot scale $k_{*}$ and the end of inflation, as well as the primordial power spectrum parameters $A_{s}, n_{s}, \alpha_{s}$, and $\alpha_{s}^{\prime}$ and the tensor-to-scalar ratio $r_{0.002}$ at the alternate scale of $k=0.002 \mathrm{Mpc}^{-1}$, are derived parameters.

UCMH constraints on inflation. - Including UCMHs and $\mathrm{PBH}$ increases the highest constrainable wavevectors in $\mathcal{P}_{\zeta}(k)$ to $k \sim 10^{18} \mathrm{Mpc}^{-1}$, significantly extending the range $\Delta \phi$ over which $V(\phi)$ can be reconstructed. While the UCMH limits on $\mathcal{P}_{\zeta}(k)$ at these small scales are orders of magnitude less severe than in the CMB range, including them has a strong effect on the higher order runnings in the spectrum. For identifying successful inflationary solutions, we require that all modes $k \leq 10^{18} \mathrm{Mpc}^{-1}$ leave the horizon during inflation, corresponding to $N_{*} \gtrsim 45$. We assume inflation can end by

\begin{tabular}{c|cc|cc|cc|cc} 
Scan \# & 0 & 1 & 2 & 3 & 4 & 5 & 6 & 7 \\
\hline $\mathrm{CMB}$ & $\checkmark$ & $\checkmark$ & $\checkmark$ & $\checkmark$ & $\checkmark$ & $\checkmark$ & $\checkmark$ & $\checkmark$ \\
$\gamma$-ray & $x$ & $x$ & $\checkmark$ & $x$ & $\checkmark$ & $x$ & $\checkmark$ & $x$ \\
Pulsar & $\boldsymbol{x}$ & $\boldsymbol{x}$ & $\boldsymbol{x}$ & $\checkmark$ & $\boldsymbol{x}$ & $\checkmark$ & $x$ & $\checkmark$ \\
$\mathrm{PBH}$ & $\boldsymbol{x}$ & $\checkmark$ & $\boldsymbol{x}$ & $\boldsymbol{x}$ & $\boldsymbol{x}$ & $\boldsymbol{x}$ & $\boldsymbol{x}$ & $\boldsymbol{x}$ \\
$z_{c}$ & - & 1000 & 500 & \multicolumn{2}{|c}{200}
\end{tabular}

TABLE I. Scan specifications. The rows show when we use $\mathrm{CMB}, \gamma$-ray UCMH, (projected) pulsar UCMH, and $\mathrm{PBH}$ data, and the redshift $z_{c}$ for UCMH formation.

\begin{tabular}{c|c|c|c} 
& Scan 0 & Scan 2 & Scan 6 \\
\hline$n_{s}$ & $0.960_{-0.011}^{+0.011}$ & $0.9650_{-0.0094}^{+0.0104}$ & $0.9650_{-0.0097}^{+0.0101}$ \\
$\alpha_{s}$ & $0.008_{-0.020}^{+0.020}$ & $-0.006_{-0.014}^{+0.014}$ & $-0.008_{-0.012}^{+0.014}$ \\
$\alpha_{s}^{\prime}$ & $0.035_{-0.029}^{+0.037}$ & $0.0025_{-0.0027}^{+0.0024}$ & $0.0005_{-0.0012}^{+0.0013}$ \\
$r_{0.002}$ & $<0.28$ & $<0.14$ & $<0.12$
\end{tabular}

TABLE II. 95.5\% CIs for the primordial parameters from the CMB-only (Scan 0), compared to conservative UCMH likelihoods (Scan 2) and the tighter constraints from UCMH with smaller $z_{c}($ Scan 6).

a hybrid transition or some other mechanism not necessarily captured in Eq. (3).

We obtain posterior probabilities for the primordial spectra and inflationary parameters using the Cosmo++ package [36] and the nested sampling code MultiNest [37] (plotted with pippi [38]). We use the Planck 2015 TT,TE,EE+lowP likelihood code [39] and the FermiLAT and pulsar UCMH likelihoods described above. We compute $\gamma$-ray and pulsar likelihoods for $10^{-6}<$ $k / \mathrm{Mpc}^{-1}<10^{18}$, applying at each $k$ the correction for the local slope of the power spectrum described in Appendix B3 of Ref. [10]; finally selecting the $k$ that produces the strongest constraint.

We use uniform priors for the cosmological parameters $\Omega_{\mathrm{b}} h^{2}, \Omega_{\mathrm{c}} h^{2}, h$, and $\tau$, and for the slow-roll parameters $\epsilon_{*}$, $\eta_{*}, \xi_{*}^{2}$, and $\omega_{*}^{3}$, with a log prior for the ratio $V_{0} / \epsilon_{*} \propto A_{s}$, matching previous analyses $[4,31]$.

We perform several scans with different assumptions (Table I). The fiducial Scan 0 uses only CMB data and agrees well with the Planck analysis [4]. Scan 1 adds PBH constraints, employing a step-function likelihood from the implementation of the limits of Ref. [15] in DarkSUSY [28], following Ref. [10]. Different scans use different UCMH parameters: $z_{c}=1000$ (Scans 2-3), $z_{c}=500$ (Scans 4-5) or $z_{c}=200$ (Scans 6-7). Scans 2, 4, and 6 add only UCMH constraints from $\gamma$-rays, while Scans 3,5 , and 7 use projected pulsar limits instead. Table II shows the $95 \%$ credible intervals (CIs) for the primordial parameters for three scans.

Fig. 1 shows the $95 \%$ CIs for $\alpha_{s}$ and $\alpha_{s}^{\prime}$. Compared to the CMB alone, using small-scale data (Scans 1-7) signif- 

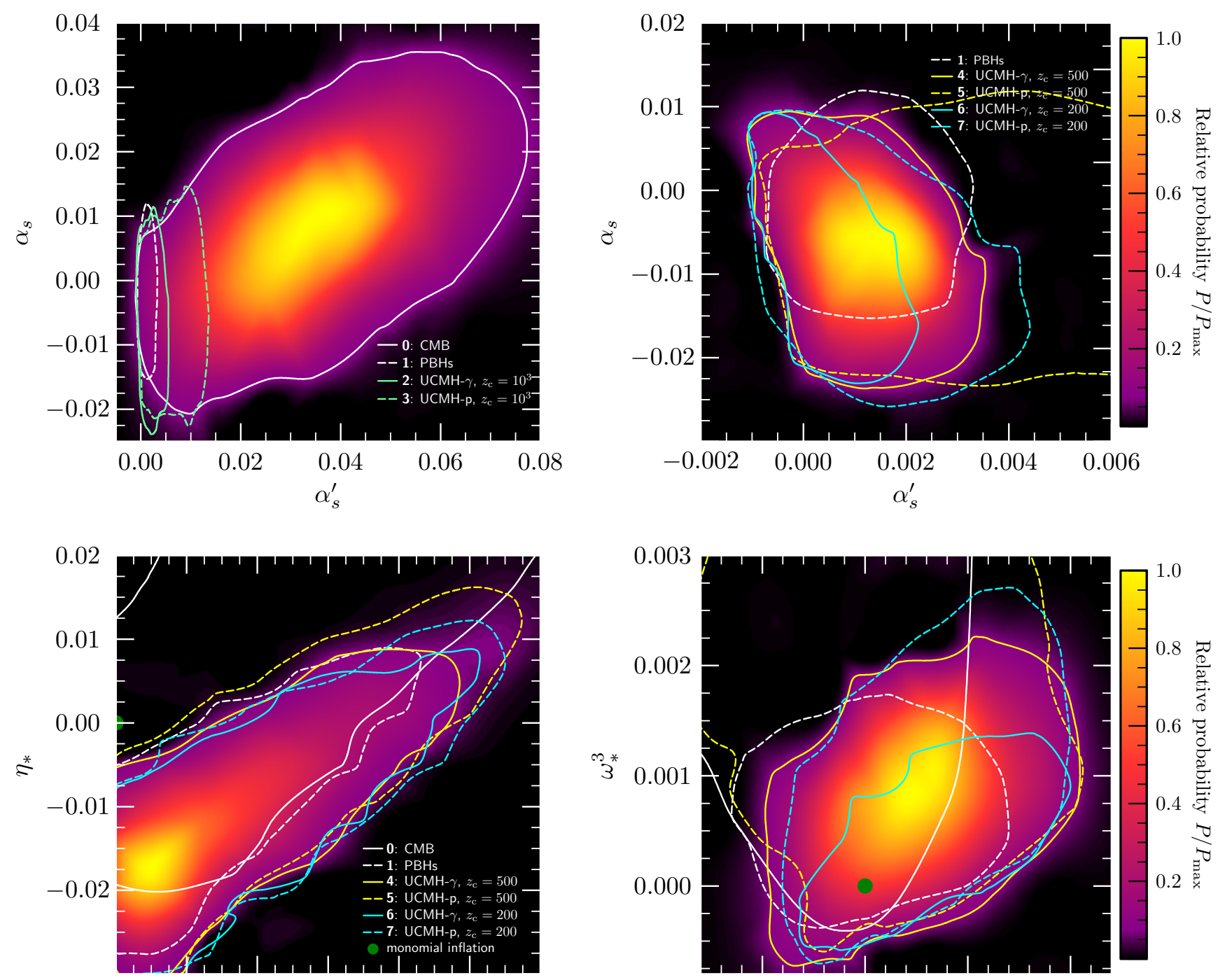

$\begin{array}{lllllll}0.000 & 0.002 & 0.004 & 0.006 & 0.008 & 0.010 & 0.012\end{array}$

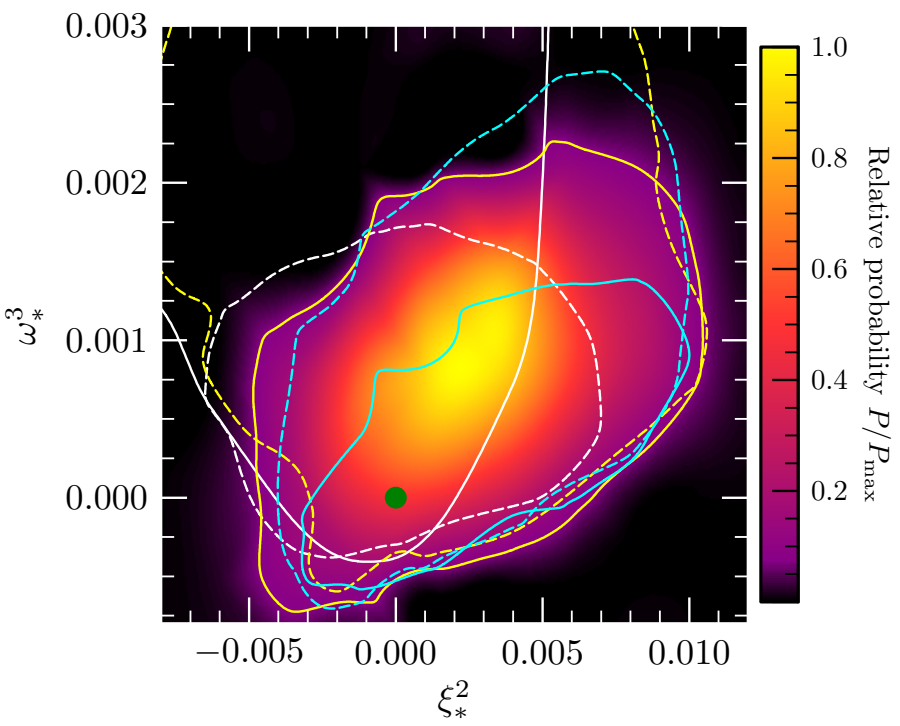

FIG. 1. (Top row) $95 \%$ credible regions (CRs) for the running $\alpha_{s} \equiv d n_{s} / d \ln k$ and the running-of-the-running $\alpha_{s}^{\prime} \equiv$ $d^{2} n_{s} / d \ln k^{2}$ of the primordial power spectrum at the pivot scale $k_{*}=0.05 \mathrm{Mpc}^{-1}$. Curves correspond to different combinations of data. 'UCMH-p' and 'UCMH- $\gamma$ ' refer to pulsar and $\gamma$-ray constraints on UCMHs, respectively. Numbers in legends refer to scans in Table I. The left and right panels are shaded by the posterior pdfs of Scans 0 and 5 , respectively. (Bottom row) 95\% CRs for the inflationary slow-roll parameters, shaded by the posterior pdf of Scan 5 . The green dot shows predictions of monomial models.

icantly tightens the credible regions on all the primordial parameters, severely limiting the shape of the inflationary potential. The $95 \% \mathrm{CI}$ for the running-of-the-running is $0 \lesssim \alpha_{s}^{\prime} \lesssim 0.05$ (Scan 2 ) or $-1 \times 10^{-3} \lesssim \alpha_{s}^{\prime} \lesssim 2 \times 10^{-3}$ (Scan 6), implying the non-observation of DM structures can robustly constrain the highest-order derivatives of $\mathcal{P}_{\zeta}(k)$. The posteriors depend strongly on $z_{\mathrm{c}}$, with much tighter constraints for $z_{c}=200$ than for $z_{c}=1000$. The UCMH likelihoods alone produce similar results to PBHs, but only become truly competitive with PBHs for $z_{c} \lesssim 500$, while the combination of PBHs and UCMHs with $z_{c}=200$ can constrain cosmological parameters much more tightly than either UCMHs or PBHs alone. More detailed knowledge of $z_{c}$ will thus be instrumental in drawing tight constraints on primordial parameters from UCMHs.

Fig. 1 also shows $95 \%$ CIs for the inflationary parameters. Comparing to the Planck results, the first two slowroll parameters have a much narrower range, $\epsilon_{*} \lesssim 0.009$ and $-0.025 \lesssim \eta_{*} \lesssim 0.01$. Scans $1-7$ prefer inflation with a lower value of the tensor-to-scalar ratio, $r \lesssim 0.13$, compared to $r \lesssim 0.28$ at $95 \%$ CI for Scan 0 , even though 
small-field inflation $\left(\epsilon_{*}<\eta_{*}\right)$ is not given equivalent weight to large-field inflation due to uniform priors on $\epsilon_{*}$ and $\eta_{*}$ [40]. Scan $6\left(z_{c}=200\right)$ has the tightest contours for the inflationary parameters, with $r \lesssim 0.12$. Including BICEP2/Keck Array CMB polarization data [41] might further reduce $r$. The higher-order slow-roll parameters $\xi_{*}^{2}$ and $\omega_{*}^{3}$ are pushed significantly toward zero by the DM constraints, mirroring the reduced range of $\alpha_{s}^{\prime}$ in Fig. 1. For comparison with some concrete models, we also show the predictions of a simple potential $V=\lambda \phi^{n}$.

We have also artificially weakened the limit on $\mathcal{P}_{\zeta}$ from UCMH constraints (not plotted) by a factor of $\sim 10$, finding little change in the results, as most scenarios predict $\alpha_{s}^{\prime}>0$ and are ruled out by even weak limits on smaller scales.

Conclusion. - Searches for UCMHs are sensitive to a wide range of amplitudes and slopes in the primordial power spectrum. UCMHs can thus be used to directly probe the preferred parameter region in inflationary models, in a way complementary to the CMB. Under conservative assumptions about the particle nature of dark matter, pulsar timing observations alone will be able to exclude a large portion of the otherwise-viable region of inflationary parameter space. If DM annihilates, nonobservation of $\gamma$-rays from DM point sources by Fermi already imposes tight constraints.

We have demonstrated for the first time that even a conservative application of the current understanding of the formation and evolution of UCMHs leads to significant limits on inflation. Future analyses would benefit from improved understanding of UCMH formation, particularly the minimum collapse redshift $z_{c}$ at which a halo can be considered a UCMH that is not significantly affected during the epoch of non-linear structure formation. Given the strength of the limits when we assume $z_{c} \lesssim 500$, urgent investigation is needed into the formation and gravitational history of the earliest bound objects in the Universe.

Acknowledgments. - LCP is supported in part by the Department of Energy under grant DESC0011114. PS is supported by STFC (ST/K00414X/1 and $\mathrm{ST} / \mathrm{N} 000838 / 1)$. HAC is supported by the University of Sydney through an Australian Postgraduate Award (APA). We acknowledge the use of the New Zealand eScience Infrastructure (NeSI) high-performance computing facilities, which are funded jointly by NeSI's collaborator institutions and through the Ministry of Business, Innovation \& Employment's Research Infrastructure programme [http://www.nesi.org.nz].

\footnotetext{
* aslanyan@berkeley.edu

† laynep@andrew.cmu.edu

‡ jenni.adams@canterbury.ac.nz
}

$\S$ torsten.bringmann@fys.uio.no

I hamish.clark@sydney.edu.au

** r.easther@auckland.ac.nz

†† geraint.lewis@sydney.edu.au

¥ p.scott@imperial.ac.uk

[1] G. Hinshaw et al. (WMAP), ApJS 208, 19 (2013), arXiv:1212.5226 [astro-ph.CO].

[2] P. A. R. Ade et al. (Planck), A\&A 571, A16 (2014), arXiv:1303.5076 [astro-ph.CO].

[3] P. A. R. Ade et al. (Planck), (2015), arXiv:1502.01589 [astro-ph.CO].

[4] P. Ade et al. (Planck), Astron.Astrophys. 571, A22 (2014), arXiv:1303.5082 [astro-ph.CO]; P. A. R. Ade et al. (Planck), (2015), arXiv:1502.02114 [astro-ph.CO].

[5] A. R. Liddle, Phys. Rev. D 49, 739 (1994), arXiv:astroph/9307020 [astro-ph].

[6] A. A. Starobinsky, Phys. Lett. B 91, 99 (1980); A. H. Guth, Phys. Rev. D 23, 347 (1981); A. D. Linde, Phys. Lett. B 108, 389 (1982); A. Albrecht and P. J. Steinhardt, Phys. Rev. Lett. 48, 1220 (1982).

[7] V. Berezinsky, V. Dokuchaev, and Y. Eroshenko, Phys. Rev. D68, 103003 (2003), arXiv:astro-ph/0301551.

[8] M. Ricotti and A. Gould, Astrophys. J. 707, 979 (2009), arXiv:0908.0735.

[9] P. Scott and S. Sivertsson, Phys. Rev. Lett. 103, 211301 (2009), [Erratum: Phys. Rev. Lett.105,119902(2010)], arXiv:0908.4082.

[10] T. Bringmann, P. Scott, and Y. Akrami, Phys. Rev. D85, 125027 (2012), arXiv:1110.2484.

[11] B. C. Lacki and J. F. Beacom, Astrophys. J. 720, L67 (2010), arXiv:1003.3466; A. S. Josan and A. M. Green, Phys. Rev. D82, 083527 (2010), arXiv:1006.4970.

[12] H. A. Clark, G. F. Lewis, and P. Scott, MNRAS 456, 1394 (2016), arXiv:1509.02938.

[13] B. J. Carr and S. W. Hawking, MNRAS 168, 399 (1974).

[14] H. V. Peiris and R. Easther, JCAP 0807, 024 (2008), arXiv:0805.2154 [astro-ph].

[15] B. J. Carr, K. Kohri, Y. Sendouda, and J. Yokoyama, Phys. Rev. D 81, 104019 (2010), arXiv:0912.5297 [astroph.CO].

[16] H. A. Clark, G. F. Lewis, and P. Scott, MNRAS 456, 1402 (2016), arXiv:1509.02941.

[17] A. D. Linde, Phys. Lett. B129, 177 (1983).

[18] J. A. Fillmore and P. Goldreich, ApJ 281, 1 (1984); E. Bertschinger, ApJS 58, 39 (1985); M. Vogelsberger, S. D. M. White, R. Mohayaee, and V. Springel, MNRAS 400, 2174 (2009), arXiv:0906.4341.

[19] V. Berezinsky, V. I. Dokuchaev, and Y. Eroshenko, Phys. Rev. D 73, 063504 (2006), astro-ph/0511494; Phys. Rev. D 77, 083519 (2008), arXiv:0712.3499; Gravitation and Cosmology 18, 57 (2012); JCAP 11, 059 (2013), arXiv:1308.6742.

[20] D. Zhang, MNRAS 418, 1850 (2011), arXiv:1011.1935; Y. Yang, X. Huang, X. Chen, and H. Zong, Phys. Rev. D 84, 043506 (2011), arXiv:1109.0156.

[21] Y.-P. Yang, X. Chen, T. Lu, and H.-S. Zong, Eur. Phys. J. Plus 126, 123 (2011), arXiv:1112.6228.

[22] That is, except for DM models with an intrinsic cutoff in the power spectrum at scales larger than probed by pulsar timing data.

[23] J. Chluba, A. L. Erickcek, and I. Ben-Dayan, Astrophys. J. 758, 76 (2012), arXiv:1203.2681 [astro-ph.CO].

[24] J. Chluba and D. Jeong, Mon. Not. Roy. Astron. Soc. 438, 2065 (2014), arXiv:1306.5751 [astro-ph.CO]. 
[25] F. Li, A. L. Erickcek, and N. M. Law, Phys. Rev. D86, 043519 (2012), arXiv:1202.1284.

[26] S. Shandera, A. L. Erickcek, P. Scott, and J. Y. Galarza, Phys. Rev. D 88, 103506 (2013), arXiv:1211.7361.

[27] Y.-P. Yang, L. Feng, X.-Y. Huang, X. Chen, T. Lu, and H.-S. Zong, JCAP 1112, 020 (2011), arXiv:1112.6229; V. S. Berezinsky, V. I. Dokuchaev, and Y. N. Eroshenko, JCAP 12, 007 (2011), arXiv:1107.2751 [astro-ph.HE].

[28] M. Anthonisen, R. Brandenberger, and P. Scott, Phys. Rev. D 92, 023521 (2015), arXiv:1504.01410.

[29] T. Bringmann, C. Kiefer, and D. Polarski, Phys. Rev. D 65, 024008 (2002), astro-ph/0109404; D. Blais, T. Bringmann, C. Kiefer, and D. Polarski, Phys. Rev. D 67, 024024 (2003), astro-ph/0206262.

[30] P. L. Nolan, A. A. Abdo, M. Ackermann, M. Ajello, A. Allafort, E. Antolini, W. B. Atwood, M. Axelsson, L. Baldini, J. Ballet, and et al., ApJS 199, 31 (2012), arXiv:1108.1435.

[31] J. Lesgourgues and W. Valkenburg, Phys. Rev. D 75, 123519 (2007), arXiv:astro-ph/0703625.

[32] P. A. R. Ade et al. (Planck), A\&A 571, A22 (2014), arXiv:1303.5082.

[33] M. J. Mortonson, H. V. Peiris, and R. Easther, Phys. Rev. D 83, 043505 (2011), arXiv:1007.4205; R. Easther and H. V. Peiris, Phys. Rev. D 85, 103533 (2012),
arXiv:1112.0326; J. Norena, C. Wagner, L. Verde, H. V. Peiris, and R. Easther, Phys. Rev. Lett. 86, 023505 (2012), arXiv:1202.0304; L. C. Price, J. Frazer, J. Xu, H. V. Peiris, and R. Easther, JCAP 1503, 005 (2015), arXiv: 1410.0685.

[34] T. S. Bunch and P. C. W. Davies, Proc. Roy. Soc. Lond. A360, 117 (1978).

[35] J. Lesgourgues, (2011), arXiv:1104.2932; D. Blas, J. Lesgourgues, and T. Tram, JCAP 1107, 034 (2011), arXiv:1104.2933.

[36] G. Aslanyan, Comp. Phys. Comm. 185, 3215 (2014), arXiv:1312.4961.

[37] F. Feroz and M. Hobson, MNRAS 384, 449 (2008), arXiv:0704.3704; F. Feroz, M. Hobson, and M. Bridges, MNRAS 398, 1601 (2009), arXiv:0809.3437; F. Feroz, M. Hobson, E. Cameron, and A. Pettitt, (2013), arXiv:1306.2144.

[38] P. Scott, Eur. Phys. J. Plus 127, 138 (2012), arXiv:1206.2245.

[39] N. Aghanim et al. (Planck), Submitted to A\&A (2015), arXiv:1507.02704 [astro-ph.CO].

[40] P. Adshead and R. Easther, JCAP 0810, 047 (2008), arXiv:0802.3898 [astro-ph].

[41] P. Ade et al. (BICEP2, Planck), Phys. Rev. Lett. 114, 101301 (2015), arXiv:1502.00612 [astro-ph.CO]. 